\title{
sciendo
}

\section{Higher-Order Vagueness and Numbers of Distinct Modalities}

\author{
Susanne Bobzien \\ University of Oxford \\ DOI: $10.2478 /$ disp-2014-0009 \\ BIBLID [0873-626X (2014) 39; pp. 131-137]
}

\begin{abstract}
This paper shows that the following common assumption is false: that in modal-logical representations of higher-order vagueness, for there to be borderline cases to borderline cases ad infinitum, the number of possible distinct modalities in a modal system must be infinite.
\end{abstract}

\section{Keywords}

Vagueness, higher-order vagueness, modalities, modal logic, KT4.

There is a not uncommon misconception regarding the relation between higher-order vagueness and the number of distinct modalities in a modal system. It is this.

(1) For a theory of higher-order vagueness to be useful towards the solution of the Sorites (by eliminating any detectable sharp boundary between non-borderline and borderline cases), it must permit the expression of radical higher-order vagueness, i.e. of borderline borderline ... borderline cases, for any number $n$ of iterations of 'borderline'. (2) An object $a$ is borderline $F$ or indeterminately $F(I F a)$ precisely if it is not determinately $F$ and not determinately not $F(\sim D F a \& \sim D \sim F a)$. (3) If higher-order vagueness is expressed by means of axiomatic — or other — systems of modal logic, the number of distinct modalities of the system must be infinite for it to be possible that there is radical higherorder vagueness.

In this paper we take issue with (3). (3) is usually rolled out as an objection to the claim that the modal system KT4 (or S4) may be suit-

Disputatio, Vol. VI, No. 39, November 2014

Received: 13/02/2014 Revised: 16/06/2014 Accepted: 19/07/2014 
able to represent higher-order vagueness. Here is a recent example:

If S4 (i.e. KT4) is the logic for absolute definiteness then there is only a finite number of modalities (in fact at most fourteen distinct modalities, see Chellas 1980, 149). Consequently, there cannot be borderline cases to borderline cases ad infinitum. (Åkerman and Greenough 2010: 287, n.37.) ${ }^{1}$

Evidently, this objection is not restricted to KT4. A modality is any sequence of the operators $\sim, \square, \diamond$. Two modalities $\Phi, \Psi$ are distinct if and only if for some $A \Phi A \leftrightarrow \Psi A$ is not a theorem. So, if we confine ourselves to familiar systems of normal modal logics and add the fact that axiom $\mathbf{T}$ seems universally accepted for logics of vagueness, then KT and KTB would be prima facie suitable, since either has infinitely many distinct modalities. On the other hand, KT4, KT5, KT4G and $\mathrm{KT}_{4} \mathrm{G}_{\mathrm{c}}$ would each be unsuitable for expressing radical higher-order vagueness, since the number of their modalities is finite.

Why would anyone think this? Åkerman and Greenough don't give much away in the paper quoted: they seem to imply that for it to be possible for there to be borderline cases to borderline cases ad infinitum (i.e. radical higher-order vagueness) there need to be infinitely many distinct modalities. Let's make the plausible assumption that this is taken to be so because each order of borderlineness needs its own distinct modality, or set of distinct modalities. For there to be borderline cases, there needs to be at least one modality; for there - also - to be borderline borderline cases, there need to be at least two, etc. Why would anyone think this? It is safe to assume that the underlying assumption is that for there to be genuine higher-order borderline cases, the extension of the borderline borderline cases must differ from that of the singly borderline cases, that of the triply borderline cases must differ from that of the doubly and the singly borderline cases, etc.

In fact, (3) from above indicates a misunderstanding of the nature of genuine higher orders in higher-order vagueness. It is a mistake to think that the number of distinct modalities in a modal system S limits the number of possible higher orders. More specifically, theorems

\footnotetext{
${ }^{1}$ This argument is different from the objections against axiom 4 that Williamson raises (1994: 157-61) and which are followed up by Greenough 2005. For some rejoinders to those objections see Bobzien 2012: 194-200, 204-210.
} 
expressing material equivalence between iterative formulas of different ranks ${ }^{2}$ in a logic of vagueness (e.g. $D A \leftrightarrow D^{2} A$ ) do not eliminate genuine higher orders. Compare epistemic logic. Assume for the sake of argument that it is logically true in some epistemic logic that I know that $A$ if and only if I know that I know that $A$. Then I would still have genuine second-order knowledge if 'I know that I know that $A$ ' is true. Exactly the same holds for higher orders of vagueness. In terms of modalized predicates, if $D F_{x}$ and $D^{\mathrm{n}} F_{x}$ are co-extensional for any $n$; or if $I F x$ and $I^{n} F x$ are co-extensional for any $n$, either way, this does not preclude that there are $a$ that are genuinely $I^{\mathrm{n}} F$. Take, for example, an epistemic interpretation of $I F a$ as ' $a$ is such that one can't tell that it is $F$ and one can't tell that it is not $F$ ', or, for short, 'a is such that one can't tell whether it is $F$ '. Assuming compositionality (and the mirror axiom $I A \leftrightarrow I \sim A$ ), $I^{2} F a$ then stands for ' $a$ is such that one can't tell whether one can tell whether it is $F$ '. Even if $I F a$ and $I^{2} F a$ are extensionally equivalent, they clearly express two different things. It is one thing for someone to be unable to tell whether $F a$, and another for someone to be unable to tell whether they are unable to tell whether Fa. The same holds for higher orders. In particular if $a$ is such that one can't tell whether one can tell ... (indefinite times) ... whether one can tell whether it is $F$, then contrary to (3) there is radical higher-order vagueness.

It is not necessary to take an epistemic interpretation. Consider instead some semantic or ontic interpretation of the indeterminacy. For instance, interpret $I F a$ as 'it is semantically indeterminate whether $\mathrm{Fa}^{\prime}$. Assuming compositionality (and the mirror axiom $I A \leftrightarrow I \sim A), I^{2} F a$ then stands for 'it is indeterminate whether it is indeterminate whether $F a^{\prime}$. Again, even if $I F x$ and $I^{2} F_{X}$ are extensionally equivalent, they clearly express two different things and contrary to (3) there is radical higher-order vagueness.

One purpose of a logic of vagueness (or indeterminacy or borderlineness) is to provide a representation of the - or certain structural properties of vagueness (or indeterminacy or borderlineness). There is nothing inherent in the notions of determinacy or indeterminacy that prohibits co-extensionality of the determinate

${ }^{2} \mathrm{DA}$ is of rank $1, \mathrm{D}^{\mathrm{n}} \mathrm{A}$ of rank $n$, etc. For a recent formal definition of modal ranks (or modal degrees) see e.g. Carnielli and Pizzi 2009: 27-8. 
and the determinately determinate, or of borderline cases and borderline borderline cases. $([\sim D F a \& \sim D \sim F a] \&[\sim D[\sim D F a \& \sim D \sim F a]$ $\& \sim D \sim[\sim D F a \& \sim D \sim F a]]$ is coherent in a system that contains $\mathrm{PC}$, MP, N, $\mathbf{K}$ and $\mathbf{T}$.) It is perfectly possible to have infinite orders of determinacy and of borderlineness with a finite number of distinct modalities. ${ }^{3}$ Note also that it follows from, and for, Williamson's account of higher-order vagueness that, if in KT4 some $A$ has secondorder vagueness, it has vagueness at every order (Williamson 1999: 132-3, 136). ${ }^{4}$

We conclude by considering two retorts which are sometimes voiced. Retort 1: "Agreed, there can be infinite orders of determinacy and borderlineness with a finite number of distinct modalities; however, this can be achieved only at the expense of introducing detectable sharp boundaries between determinate cases and borderline cases." One can see how someone might get this idea by examining KT5 and KT4 and coming to the conclusion that neither is suitable for eliminating sharp boundaries. Given (2) and modal axioms 4 and 5, KT5 provides, for a vague predicate F, only (i) determinate cases of $F$, (ii) determinate indeterminate cases of $F$ and (iii) determinate cases of $\sim F$. This suggests sharp borders into and out of the borderline zone. And Williamson (1999: 134) shows that with his own formal characterization of higher-order vagueness, system S5 is the weakest extension of KT that would permit vagueness and forbid higher-order vagueness. As for KT4, it may appear to lead to a

\footnotetext{
${ }^{3}$ This holds regardless of whether higher-order vagueness is defined (i) as ' $A$ is $n^{\text {th }}$-order vague if $I^{n} A$ (and $F$ is $n^{\text {th }}$-order vague if $\exists_{X} I^{n} F x$ )'; or (ii) with Williamson (1999: 132) as "[w]e have a first-order classification of states of affairs according to whether $A$ or $\sim A$ holds. Vagueness in the first-order classification is first-order vagueness in $A$. [ ...] we have an $(n+1)^{\text {th }}$-order classification according to whether members of the $n$ th-order classification definitely hold, definitely fail to hold or are borderline cases. Vagueness in the $n$ th-order classification is $n$ th-order vagueness in A"; or (iii) in any other way directly based on (2).

4 In Williamson's account (see previous note), $\sim D D^{n} A \& \sim D \sim D^{n} A$ with $n \geq 0$ is a sufficient condition for $(n+1)^{\text {th }}$ order vagueness. By $D A \leftrightarrow D^{\mathrm{n}} A$ for $n \geq 1$ in KT4 we get (i) $\sim D D A \& \sim D \sim D A \rightarrow \sim D D^{n} A \& \sim D \sim D^{n} A$. We get (ii) $\sim D D A \& \sim D \sim D A \rightarrow \sim D A \& \sim D \sim A$ by the KT4 theorems (iii) $D A \rightarrow D D A$ and (iv) $\sim D \sim D A \rightarrow \sim D \sim A$ : (iii) together with the contraposition of (iv) provides $D A \vee D \sim A \rightarrow D D A \vee D \sim D A$, which by contraposition and DeMorgan gives (ii). (ii) covers the case of $n=0$ and (i) covers the cases with $n>0$.
} 
sharp border from the $n$ times determinate cases $\left(D^{\mathrm{n}} F\right)$ to the $n$ times borderline cases $\left(I^{\mathrm{n}} F\right)$ at the beginning of some assumed borderline zone and for indefinite $n$. However, in both cases the argument is not that the extensions of the borderline, and the borderline borderline, cases, etc., are co-extensive. Rather, for KT5 the argument is that there is a sharp boundary between the determinately determinate cases and the determinate borderline cases; and for KT4 it would be that there is a sharp boundary between the cases that are $D^{\mathrm{n}} F$ and the borderline cases that are $I^{\mathrm{n}} F$. Thus, even though KT5 and KT4 may have been shown to be unsuitable for avoiding a sharp boundary, it has not been shown that this is so because the number of their distinct modalities is finite. More importantly, system $\mathrm{KT}_{4} \mathrm{G}_{\mathrm{c}}$ or $\mathrm{S} 4 \mathrm{M}$, which adds axiom $\mathbf{G}_{\mathbf{c}}(\square \diamond A \rightarrow \diamond \square A)$ to $\mathrm{KT} 4$, and which has only a measly eight distinct modalities, both preserves higher-order vagueness and complies with the intuition that there are no detectable sharp boundaries between borderline and non-borderline cases. In its determinacy version it has both $D A \leftrightarrow D^{2} A$ and $I A \leftrightarrow I^{2} A$ as theorems and thus introduces infinite orders of both determinacy and indeterminacy (or borderlineness). At the same time $\mathrm{KT}_{4} \mathrm{G}_{\mathrm{c}}$ defines a logic of determinacy that has as one of its inherent features that no sharp boundary between the borderline cases and the non-borderline cases can be determined. ${ }^{5}$

Retort 2: "By $a$ being borderline $F$ we don't just mean $\sim D F a \& \sim D \sim F a$. The borderline cases also have to be between the determinate cases." This is, of course, changing the rules halfway through the game. Instead of the standard modal account of borderlineness (2) from above, we now have something like this (we offer a charitable version), with $B L F a$ for $a$ is borderline $F$ :

(4) BLFa if and only if $[\sim D F a \& \sim D \sim F a] \& a$ is between the things that satisfy $D F$ and the things that satisfy $D \sim F$.

(5) $\mathrm{BL}^{2} \mathrm{~F} a$ if and only if $[\sim D B L F a \& \sim D \sim B L F a] \& a$ is between the things that satisfy $D B L F$ and the things that satisfy $D \sim B L F$.

${ }^{5}$ Bobzien 2010 provides an extended argument for the compatibility of radical higher-order vagueness with axiom 4 and with the characteristic axiom of $\mathrm{KT}_{4} \mathrm{G}_{\mathrm{c}}$. 
It is accounts of borderlineness along the lines of (4) and (5) which open the door for the so-called higher-order vagueness paradoxes. ${ }^{6}$ We believe that such accounts and the ensuing presumed paradoxes are the result of a confusion between higher-order vagueness and the distribution of the objects of a Sorites series into extensionally nonoverlapping categories. ${ }^{7}$ But even with (4) and (5), the numbers of higher orders do not depend on the numbers of distinct modalities: with a sufficiently fine-grained Sorites series nothing prevents there from being more than, say, fourteen higher orders. In any event, we set out to show that, given (2), (3) is false; i.e. that, given (2), there cannot be "borderline cases to borderline cases ad infinitum", even with a finite number of distinct modalities such as in KT4. And this we have shown. ${ }^{8}$

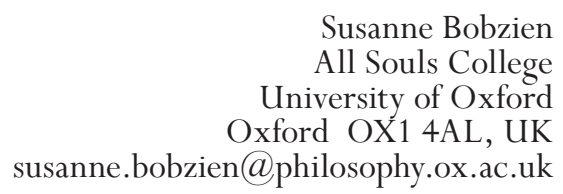

\section{References}

Åkerman, Jonas and Greenough, Patrick. 2010. Hold the Context Fixed: Vagueness Still Remains. In Cuts and Clouds: Vagueness, its Nature, and its Logic. Edited by Dietz, Richard and Moruzzi, Sebastian. Oxford: Oxford University Press, 275-88.

Bobzien, Susanne. 2010. Higher-order Vagueness, Radical Unclarity, and Absolute Agnosticism. Philosophers' Imprint 10: 1-30.

Bobzien, Susanne. 2012. If it's Clear, then it's Clear that it's Clear, or is it? — Higher-order Vagueness and the S4 Axiom. In Episteme, etc. Edited by Katerina Ierodiakonou and Benjamin Morison. Oxford: Oxford University Press, 189-212.

Bobzien, Susanne. 2013. Higher-order Vagueness and Borderline Nestings — a Persistent Confusion. Analytic Philosophy 54: 1-43.

${ }^{6}$ See Fara 2003: 196-200, Sainsbury 1991: 167-70, Shapiro 2005: 147-51, Wright 1992: 129-33, 137 and Greenough 2005: 182-3 for different versions of this type of presumed paradox.

${ }^{7}$ For a detailed account of this confusion see Bobzien 2013.

${ }^{8}$ Thanks to Nicholas Denyer and to an anonymous referee from Disputatio for helpful comments. 
Carnielli, Walter and Pizzi, Claudio. 2009. Modalities and Multimodalities. New York: Springer.

Chellas, Brian F. 1980. Modal Logic: An Introduction. Cambridge: Cambridge University Press.

Fara, Delia Graff. 2003. Gap principles, penumbral consequence and infinitely higher-order vagueness. In Liars and Heaps: New Essays on Paradox. Edited by J. C. Beall.Oxford: Oxford University Press, 195-222. Originally published under the name 'Delia Graff'.

Greenough, Patrick. 2005. Contextualism about Vagueness and Higher-Order Vagueness. Proceedings of the Aristotelian Society (suppl) 105: 167-90.

Sainsbury, Mark. 1991. Is There Higher-Order Vagueness? Philosophical Quarterly 41: $167-82$

Shapiro, Stewart. 2005. Context, Conversation, and so-called 'Higher-Order Vagueness'. Proceedings of the Aristotelian Society (suppl) 105: 147-65.

Williamson, Timothy. 1994. Vagueness. London: Rougledge.

Williamson, Timothy. 1999. On the structure of higher-order vagueness. Mind 108: 127-142.

Wright, Crispin. 1992. Is Higher-Order Vagueness Coherent? Analysis 52: 129-39. 